\title{
Avaliação do conhecimento sobre a pandemia Covid-19 entre estudantes de graduação do interior do estado Rio Grande do Norte
}

\author{
Talita Araujo de Souza \\ Mestre em Saúde Coletiva (UFRN). Doutoranda em Ciências da Saúde (UFRN). \\ $\triangle$ tulio.quirino@recife.pe.gov.br \\ Sávio Marcelino Gomes \\ Mestre em Saúde Coletiva (UFRN). Doutoranda em Saúde Coletiva (UFRN) \\ $\triangle$ svgomes77@gmail.com \\ Maria Helena Rodrigues Galvão \\ Mestre em Saúde Coletiva (UFRN). Doutoranda em Saúde Coletiva (UFRN) \\ $\triangle$ mhrgalvao@gmail.com \\ Isabelle Ribeiro Barbosa \\ Doutora em Saúde Coletiva (UFRN). Docente do Programa de Pós-Graduação em Saúde Coletiva (UFRN) \\ $\triangle$ isabelleribeiro68@hotmail.com
}

Recebido em 7 de maio de 2020

Aceito em 2 de julho de 2020

\begin{abstract}
Resumo:
Esse estudo tem por objetivo avaliar o conhecimento sobre a pandemia do Covid-19 entre estudantes de graduação da área da saúde. Configura-se como um estudo transversal, do tipo pesquisa de opinião pública, realizado com estudantes dos cursos de Enfermagem, Nutrição, Psicologia e Fisioterapia do campus da UFRN no município de Santa Cruz. A amostra foi composta por os 282 estudantes, a coleta ocorreu por formulários eletrônicos no Google forms $s^{\circledast}$, divulgado através das mídias sociais (WhatsApp, Facebook e Instagram). Foi realizada análise bivariada utilizando o teste Qui-quadrado e exato de Fischer. Os estudantes possuem um conhecimento adequado acerca da doença, a maior parte disse ter recebido orientação, conhece a paramentação adequada para prevenção, acha que sua saúde mental está prejudicada e acredita que a suspensão das aulas trará impacto no seu desenvolvimento acadêmico. A média de acertos sobre os aspectos biológicos foi de 6,57, enquanto dos aspectos epidemiológicos foi de 3,10. Não houve diferença significativa quando comparado entre os cursos. 0 conhecimento dos estudantes sobre o Covid-19 foi considerado regular. Esses resultados apontam para a necessidade das atualizações diárias sobre a doença entre os estudantes.
\end{abstract}

Palavras-chave: Covid-19, Coronavírus, Estudantes, Graduação, Conhecimento.

\section{Evaluation of knowledge about the covid-19 pandemic among undergraduate students from the hinterland of the state Rio Grande do Norte}

\begin{abstract}
:
This study aims to It was intended to evaluate the knowledge about the Covid-19 pandemic among undergraduate students in the health area. It is configured as a cross-sectional study, typified as public opinion research, carried out with students from the Nursing, Nutrition, Psychology and Physiotherapy courses at the UFRN campus in the town of Santa Cruz. The sample was composed by 282 students, and the collection took place using electronic forms on Google forms ${ }^{\oplus}$, disseminated through social media (WhatsApp,
\end{abstract}


Facebook and Instagram). Bivariate analysis was performed using the Chi-square test and Fischer's exact test. The students have appropriate knowledge about this disease, most of them said they had received guidance, they know the proper attire for prevention, they think their mental health is impaired and they believe that the suspension of classes will entail an impact on their academic development. The average rate of correct answers on the biological aspects was 6.57, while on the epidemiological aspects it was 3.10. There was no significant difference when the courses were compared. Students' knowledge about Covid19 was considered regular. These results highlight the need for daily updates on the disease among students.

Keywords: Covid-19, Coronavirus, Students, Undergraduate Courses, Knowledge.

\section{Evaluación del conocimiento sobre la pandemia covid-19 entre estudiantes de pregrado del interior del estado de Rio Grande do Norte}

\section{Resumen:}

Este estudio tiene como objetivo evaluar el conocimiento sobre la pandemia del Covid-19 entre estudiantes de pregrado del área de la salud. Se configura como un estudio transversal, tipificado como investigación de opinión pública, realizado con estudiantes de los cursos de Enfermería, Nutrición, Psicología y Fisioterapia del campus de la UFRN en el ayuntamiento de Santa Cruz. La muestra se compuso de 282 estudiantes, y la recolección tuvo lugar mediante formularios electrónicos en el Google forms $s^{\circledast}$, difundidos a través de los medios sociales (WhatsApp, Facebook e Instagram). El análisis bivariado se realizó utilizando la prueba de Chi-cuadrado y la prueba exacta de Fischer. Los estudiantes tienen un conocimiento adecuado sobre la enfermedad, donde la mayoría declararon que habían recibido orientación, conocen la vestimenta adecuada para la prevención, creen que su salud mental está deteriorada y piensan que la suspensión de las clases tendrá un impacto en su desarrollo académico. El promedio de respuestas correctas sobre los aspectos biológicos fue de 6,57, mientras que sobre los aspectos epidemiológicos fue de 3,10. No hubo diferencias significativas cuando se comparan los cursos. El conocimiento de los estudiantes sobre el Covid19 se consideró regular. Estos resultados señalan la necesidad de actualizaciones diarias sobre la enfermedad entre los estudiantes.

Palabras clave: Covid-19, Coronavirus, Estudiantes, Pregrado, Conocimiento.

\section{INTRODUÇÃO}

Em dezembro 2019, a Organização Mundial da Saúde (OMS) foi notificada sobre a ocorrência de um surto de pneumonia de forma mais grave, na cidade de Wuhan, província de Hubei, República Popular da China. Cientistas mobilizaram-se e logo foi identificado o agente etiológico, um novo coronavírus: SARS-COV-2 (ZHU et al., 2020).

Infecções por coronavírus (CoV) são vírus respiratórios emergentes e provocam infecção que variam do resfriado comum à síndrome respiratória aguda grave (SARS) (YIN; WUNDERINK, 2018). CoV é um caracterizado como um zoonótico patógeno que pode ser transmitido através da interação animal-humano e humano-humano (LI et al., 2020). Múltiplo surtos epidêmicos ocorreram durante 2002 (SARS), com média de 800 mortes, e 2012 (no Oriente Médio Síndrome respiratória: MERS-CoV), com 860 óbitos (YIN; WUNDERINK, 2018). 
Aproximadamente oito anos após a epidemia de MERS-CoV, o atual surto de novo coronavírus (COVID-19) emergiu como uma pandemia (LAI et al., 2020).

No dia 30 de janeiro de 2020, a OMS declarou que o COVID-19 era uma emergência de saúde pública preocupação internacional (WHO, 2020). Ao passar dos dias, na primeira semana de março, um devastador número de casos novos foram notificados globalmente e o COVID-19 emergiu como uma pandemia. A partir de 12 de março de 2020, mais de 125.000 casos confirmados em 118 países e mais de 4600 mortes foram relatados. O COVID-19 é transmitido por transmissão homem-a-homem através de gotículas e contato direto e tem um período de incubação de 2-14 dias (WHO, 2020). Até o momento, nenhum tratamento antiviral ou vacina foi explicitamente recomendado para COVID-19. Dessa forma, a aplicação de medidas preventivas para controlar a infecção por COVID-19 é a intervenção mais efetiva até o momento (BHAGAVATHULA et al., 2020).

No Brasil, em 28 de janeiro, foi publicado o primeiro Boletim Epidemiológico, um guia de vigilância epidemiológica e um Plano de Contingência Nacional para a COVID-19, que teve como objetivo orientar o Sistema Nacional de Vigilância e a rede de serviços do Sistema Único de Saúde (SUS) para atuarem na identificação da COVID-19, a fim de mitigar os riscos de transmissão sustentada e aparecimento de casos graves e mortes subsequentes (CRODA et al., 2020).

O primeiro caso de COVID-19 no Brasil foi confirmado em 26 de fevereiro de 2020, e até 03 de março, 488 casos suspeitos foram relatados, 2 foram confirmados e 240 foram descartados no Brasil, sem evidências de transmissão local. Os dois primeiros casos confirmados ocorreram em homens residentes na cidade de São Paulo, SP, que haviam retornado de uma viagem na Itália (CRODA et al., 2020). Atualmente, a doença tem mostrado um significativo crescimento no país, e até 27 de abril de 2020, haviam 58.509 casos notificados e 4016 mortes registradas (WHO, 2020).

De acordo com o boletim epidemiológico do Rio Grande do Norte do dia 28 de abril de 2020, o estado possui até o momento 857 casos confirmados, 4.626 suspeitos, 3.002 descartados e 48 óbitos. Existe uma tendência de aumento nas notificações ao longo da 
semana, predominando o sexo feminino e a faixa etária de 30 a 39 anos (SECRETARIA DE SAÚDE PÚBLICA DO ESTADO DO RIO GRANDE DO NORTE, 2020).

A propagação do COVID-19 tem exigido avaliações rápidas do conhecimento e percepções das pessoas acerca desta infecção (GELDSETZER, 2020). Quando avaliados os surtos de doenças infecciosas que se deslocam rapidamente, como a COVID-19, a avaliação do conhecimento e das percepções de populações específicas deve ser realizada em um curto espaço de tempo para que os resultados sejam informativos à resposta da saúde pública (GELDSETZER, 2020).

Dessa forma, pesquisas on-line rápidas, que exigem recursos humanos mínimos e podem atingir um grande número de entrevistados em um curto espaço de tempo, podem ser uma ferramenta valiosa para avaliar (e monitorar ao longo do tempo) o conhecimento e percepções de uma doença infecciosa no meio de um surto (ZHONG et al., 2020). Nesse sentido, torna-se importante conhecer o grau de conhecimento de estudantes de graduação da área da saúde sobre a pandemia do Covid-19, principalmente considerando que os estudantes da saúde como futuros profissionais também poderão ter que atuar com situações semelhantes.

Sendo assim, esse estudo se propõe a avaliar o conhecimento sobre a pandemia do Covid-19 entre estudantes de graduação da área da saúde de uma instituição pública de ensino no interior do Rio Grande do Norte, Brasil.

\section{METODOLOGIA}

Trata-se de um estudo transversal, do tipo pesquisa de opinião pública. De acordo com a resolução no 510/2018 do Ministério da Saúde, a pesquisa de opinião pública é caracterizada por avaliar preferências e sentidos que se atribuem a diversos temas, sem conferir identificação aos sujeitos pesquisados. Por essas características, a resolução exime este tipo de pesquisa da avaliação pelo Comitê de Ética em Pesquisa (CEP). Todas as etapas do presente estudo estão de acordo com a referida resolução.

Foi realizada com estudantes da Faculdade de Ciências da Saúde do Trairí - FACISA, campi de expansão da Universidade Federal do Rio Grande do Norte (UFRN), localizado no 
município de Santa Cruz, situado a 120 km de distância da capital do estado do Rio Grande do Norte. A população estimada para o município pelo Instituto Brasileiro de Geografia e Estatística (IBGE) para o ano de 2019 foi de 39.674 habitantes, com IDH de 0,635. O município é sede da V Unidade Regional de Saúde Pública - V URSAP, parte integrante à estrutura da Secretaria Estadual de Saúde Pública - SESAP.

A população do estudo foi composta por estudantes ativos dos cursos da FACISA/UFRN, sendo estes Enfermagem, Fisioterapia, Nutrição e Psicologia. O tamanho da amostra foi calculada através da fórmula $n=[\operatorname{DEFF} * N p(1-p)] /\left[\left(d^{2} / Z^{2}{ }_{1-\alpha / 2} *(N-1)+p *(1-\right.\right.$ p)), por meio da plataforma Open Epi, assumindo a prevalência de 50\% pelo desfecho ser desconhecido, com margem de erro absoluta de 5\%, considerando intervalo de $95 \%$ de confiança e uma taxa de não-resposta de 10\%. A amostra resultante foi de 282 estudantes.

Foi realizada amostragem estratificada e não probabilística, de acordo com as proporções de estudantes matriculados em cada curso, nos quais os 282 estudantes foram divididos em 57 estudantes de enfermagem, 71 de fisioterapia, 73 de nutrição e 81 de psicologia.

A coleta de informações foi realizada através de formulários eletrônicos no Google forms $^{\circledast}$, divulgado através de e-mail dos estudantes e redes sociais como Whatsapp, Facebook e Instagram. Ao alcançar o número amostral de estudantes em cada categoria o formulário foi encerrado.

As variáveis utilizadas foram gênero (masculino ou feminino), curso (enfermagem, fisioterapia, nutrição ou psicologia), meios de informação mais utilizados sobre Coronavírus (internet, redes sociais, artigos científicos, amigos e familiares e outros), bem como variáveis relacionadas a realização de orientações ou treinamento, rendimento acadêmico, saúde mental e os aspectos biológicos, clínicos e epidemiológicos da doença, todas dicotômicas (discordo ou concordo).

Foi realizada análise descritiva das variáveis, através de frequência absoluta e relativa, bem como análises bivariadas em relação ao curso. As variáveis relacionadas aos aspectos biológicos, clínicos e epidemiológicos da doença foram pontuados, criando um score, o qual foi avaliado quanto a média, desvio padrão, moda, mediana, valor mínimo e máximo. Por fim, 
foi realizada uma nova bivariada considerando a divisão dos estudantes entre aqueles que responderam corretamente todas as questões e aqueles que não o fizeram. Os cruzamentos foram realizados através do teste Qui-quadrado de Pearson e Teste exato de Fischer, quando aplicável. Todas as análises foram realizadas no software Epi Info ${ }^{\mathrm{TM}}$.

\section{RESULTADOS}

Sobre o perfil dos estudantes respondentes, observou-se que a maior parte $(78,7 \%)$ era do sexo feminino. O curso com maior percentual de respondentes foi o de Psicologia, representado 30,9\% da amostra. 0 principal meio de informação utilizado por estudantes para atualização acerca da COVID-19 foi a internet, 91,7\% dos estudantes utilizaram esse meio para busca de informações. Os artigos científicos só foram utilizados por $42,7 \%$ dos estudantes, sendo as redes sociais mais utilizadas para essa finalidade que artigos em periódicos científicos, sendo utilizada por $65,6 \%$ dos estudantes (Tabela 1 ).

A maior parte dos estudantes, $68,8 \%$, referiu ter recebido algum tipo de treinamento ou orientação sobre prevenção e controle de infecção pelo Coronavírus. Apesar disto, a maioria dos estudantes $(80,6 \%)$ referiu ter conhecimento acerca da paramentação completa para evitar a infecção pelo Coronavírus. Para $62,1 \%$ dos estudantes, a interrupção das atividades terá influência sobre o seu rendimento acadêmico. Sobre a saúde mental no momento de pandemia, a maior parte dos estudantes $(69,4 \%)$ referiu sentir prejuízos em sua saúde mental.

Tabela 1 - Caracterização dos respondentes e percepção quanto aos aspectos relacionados à COVID-19 por estudantes universitários dos cursos de saúde de uma Faculdade de Ciências da Saúde no interior do Nordeste, Brasil, 2020.

\begin{tabular}{lccc}
\hline Variáveis & $\mathbf{n}$ & $\mathbf{\%}$ \\
\hline Gênero & 247 & 78,7 \\
Feminino & 67 & 21,3 \\
Masculino & & \\
Curso & 58 & 18,5
\end{tabular}


Fisioterapia

78

Nutrição

Psicologia

Meios de informação mais utilizados sobre coronavírus

Internet

Redes sociais

Artigos científicos

Amigos e familiares

Outros

Recebi algum tipo de treinamento ou orientação sobre prevenção e controle de infecção pelo coronavírus

Discordo

Concordo

Conheço sobre paramentação correta de EPI para evitar infecção pelo coronavírus

Discordo

Concordo

Acredito que a interrupção das atividades acadêmicas terá impacto direto no meu rendimento acadêmico

Discordo

Concordo

Sinto que a minha saúde mental está prejudicada com a atual pandemia

Discordo

Concordo
288

8125,8

$97 \quad 30,9$

91,7

$206 \quad 65,6$

$134 \quad 42,7$

6922,0

$96 \quad 30,6$

98

31,2

$216 \quad 68,8$

$61 \quad 19,4$

$253 \quad 80,6$

$119 \quad 37,9$

$195 \quad 62,1$

Fonte: Própria.

Com relação ao conhecimento sobre aspectos biológicos e o curso clínico da doença, $96,2 \%$ dos estudantes referiram conhecer o nome científico do coronavírus. A maior parte dos estudantes $(83,8 \%)$ concordou com a premissa de conhecer a origem do coronavírus. A extensa maioria dos estudantes concorda que conhece os sinais e sintomas comuns da doença causada pela infecção por coronavírus. 89,8 dos estudantes também discorda que a presença de febre, gripe e tosse é significa que a pessoa está infectada. A maior parte dos estudantes 
discorda da possibilidade de contágio por animais de estimação (93,9\%). Sobre a cura para a COVID-19, 96,5\% dos estudantes concordam que ainda não há cura eficaz para a doença, havendo apenas tratamento sintomático. A maior parte dos estudantes $(98,4 \%)$ também concorda quem nem todas as pessoas evoluirão para casos graves da doença (Tabela 2).

Sobre os aspectos epidemiológicos, a maior parte dos estudantes (96,8\%) concorda de que já houveram infecções virais pandêmicas no passado, e concorda que o Brasil possui política e diretrizes de controle de infecção pelo Coronavírus (56,1\%). A maioria dos estudantes $(69,7 \%)$ acredita que é possível parar a propagação do vírus no Brasil, e concorda que a estratégia mais eficaz para evitar a propagação do vírus é o isolamento social (99,7\%).

Tabela 2 - Percepção dos estudantes universitários dos cursos de saúde de uma Faculdade de Ciências da Saúde no interior do Nordeste quanto ao conhecimento de aspectos biológicos, clínicos e epidemiológicos relacionados à pandemia de COVID-19, Brasil, 2020.

\begin{tabular}{|c|c|c|}
\hline Variável & $\mathbf{n}$ & $\%$ \\
\hline \multicolumn{3}{|c|}{ Aspectos biológicos e curso clínico da doença } \\
\hline \multicolumn{3}{|c|}{ Conheço o nome científico do Coronavírus } \\
\hline Discordo & 12 & 3,8 \\
\hline Concordo & 302 & 96,2 \\
\hline \multicolumn{3}{|c|}{ Conheço a origem do Coronavírus } \\
\hline Discordo & 51 & 16,2 \\
\hline Concordo & 263 & 83,8 \\
\hline \multicolumn{3}{|c|}{$\begin{array}{l}\text { Tenho conhecimento dos sinais e sintomas comuns do } \\
\text { coronavírus }\end{array}$} \\
\hline Discordo & 2 & 0,6 \\
\hline Concordo & 312 & 99,4 \\
\hline \multicolumn{3}{|c|}{ Ter febre, gripe e tosse significa que a pessoa está infectada } \\
\hline Discordo & 282 & 89,8 \\
\hline Concordo & 32 & 10,2 \\
\hline \multicolumn{3}{|c|}{ É possível adquirir coronavírus de animais de estimação } \\
\hline Discordo & 295 & 93,9 \\
\hline Concordo & 19 & 6,1 \\
\hline
\end{tabular}


Atualmente, não existe uma cura eficaz para o COVID-19, mas o tratamento sintomático e de suporte precoce pode ajudar a maioria os pacientes a se recuperar da infecção

$\begin{array}{lcc}\text { Discordo } & 11 & 3,5 \\ \text { Concordo } & 303 & 96,5\end{array}$

Nem todas as pessoas com Coronavírus evoluirão para casos graves. Os idosos, as pessoas que têm doenças crônicas e os obesos têm maior probabilidade de desenvolverem quadros graves

Discordo $5 \quad 1,6$

Concordo 309

98,4 Aspectos epidemiológicos

Já existiram outras infecções virais pandêmicas no passado

Discordo

Concordo

O Brasil possui política e diretrizes de controle de infecção pelo Coronavírus

Discordo

Concordo

Podemos parar a propagação viral no Brasil

Discordo

Concordo

Acredito que isolamento social é a medida mais eficaz contra a rápida expansão da doença

Discordo

Concordo

Fonte: Própria.

A Tabela 3 apresenta a distribuição, por curso de graduação, das respostas dos estudantes acerca da COVID-19. Observou-se que para a maioria das variáveis do questionário não houveram diferenças entre as proporções entre os cursos de graduação da instituição. Observaram-se diferenças estatisticamente significantes na distribuição entre os curso para a variável "Recebi algum tipo de treinamento ou orientação sobre prevenção e controle de 
infecção pelo coronavírus", onde o curso de Fisioterapia apresentou um maior percentual de estudantes que receberam algum tipo de capacitação $(78,2 \%)$, enquanto o curso de Nutrição apresentou menor percentual (61,7\%). Também foram observadas diferenças estatisticamente significantes entre os cursos com relação ao conhecimento sobre paramentação correta para evitar a infecção pelo coronavírus, onde o curso de Nutrição apresentou maior percentual de estudantes que conhecem sobre a paramentação adequada (90,1\%), enquanto entre os estudantes do curso de psicologia apenas $64,9 \%$ relataram possuir tal conhecimento.

Tabela 3 - Análise do conhecimento dos estudantes acerca dos aspectos associados à COVID-19, por curso de graduação, através do teste do Qui-quadrado de Pearson. 2020.

\begin{tabular}{cc|c|c|c}
\hline \multicolumn{4}{c}{ Curso } & valor \\
\cline { 2 - 5 } Enfermagem & Fisioterapia & Nutrição & Psicologia & de p \\
n(\%) & n(\%) & n(\%) & n(\%) \\
\hline
\end{tabular}

\begin{tabular}{lrr}
\hline Conheço & o & nome \\
científico & do
\end{tabular}

\section{Coronavírus}

$\begin{array}{lccccc}\text { Discordo } & 1(1,7) & 3(3,8) & 5(6,2) & 3(3,1) & 0,562 \\ \text { Concordo } & 57(98,3) & 75(96,2) & 76(93,8) & 94(96,9) & \end{array}$

Conheço a origem do

Coronavírus

Discordo

$17(21,8)$

$10(12,3)$

15(15,5)

Concordo

49(84,5)

$61(78,2)$

$71(87,7)$

0,434

Tenho conhecimento dos

sinais e sintomas comuns

do coronavírus
Discordo
Concordo
Ter febre, gripe e tosse
significa que a pessoa

0

0

$58(100,0) \quad 78(100,0)$

$2(2,5)$

$82(84,5)$

está infectada 
Discordo

Concordo

É possível adquirir

coronavírus de animais

de estimação

Discordo

Concordo

Já existiram outras infecções virais

pandêmicas no passado

Discordo

Concordo

Podemos parar a propagação viral no

Brasil

Discordo

Concordo

O Brasil possui política e diretrizes de controle de infecção pelo

Coronavírus

Discordo
Concordo

Recebi algum tipo de treinamento ou orientação sobre prevenção e controle de infecção pelo coronavírus

Discordo
Concordo

$$
\begin{array}{cc}
57(98,3) & 70(89,7) \\
1(1,7) & 8(10,3)
\end{array}
$$$$
69(85,2)
$$$$
12(14,8)
$$$$
86(88,7)
$$$$
11(11,3)
$$

$\begin{array}{ccccc}51(87,9) & 74(94,9) & 78(96,3) & 92(94,8) & \\ 7(12,1) & 4(5,1) & 3(3,7) & 5(5,2) & 0,192\end{array}$


Conheço

sobre

paramentação correta de

EPI para evitar infecção

pelo coronavírus

Discordo

Concordo

Acredito que a

interrupção das

atividades acadêmicas

terá impacto direto no

meu rendimento

acadêmico

Discordo

Concordo

Sinto que minha saúde mental está prejudicada com a atual pandemia

Discordo

Concordo

Acredito que isolamento social é a medida mais eficaz contra a rápida expansão da doença

Discordo

Concordo

Atualmente, não existe uma cura eficaz para o COVID-19, mas o tratamento sintomático e de suporte precoce pode ajudar a maioria os

\begin{tabular}{|c|c|c|c|}
\hline $7(12,1)$ & $12(15,4)$ & $8(9,9)$ & $34(35,1)$ \\
\hline $51(87,9)$ & $66(84,6)$ & $73(90,1)$ & $63(64,9)$ \\
\hline
\end{tabular}

$22(39,7) \quad 24(30,8) \quad 28(34,6) \quad 44(45,4)$

$35(60,3) \quad 54(69,2) \quad 53(65,4) \quad 53(54,6)$

0,218

$\begin{array}{lllll}22(37,9) & 22(28,2) & 25(30,9) & 27(27,8) & \\ 36(62,1) & 56(71,8) & 56(69,1) & 70(72,2) & 0,566\end{array}$
0
0
0
$1(1,0)$

$58(100,0)$

$78(100,0)$

$81(100,0)$

0,523 


\begin{abstract}
pacientes a se recuperar
da infecção

Discordo

Concordo

Nem todas as pessoas

com Coronavírus

evoluirão para casos

graves. Os idosos, as

pessoas que têm doenças

crônicas e os obesos têm

maior probabilidade de

desenvolverem quadros

graves
\end{abstract}

$1(1,3)$

$57(98,3)$

$77(97,7)$
$4(4,1)$

$93(95,9)$

$\begin{array}{lccccc}\text { Discordo } & 2 & 1 & 0 & 2 & 0,428 \\ \text { Concordo } & 56(96,6) & 77(98,7) & 81(100,0) & 97(97,9) & \end{array}$

Fonte: Própria.

Com relação a pontuação da dimensão relacionada aos aspectos biológicos e curso clínico da doença, que é composta por 7 quesitos, observou-se que média de pontos entre os estudantes foi de 6,57 (DP=0,66), a pontuação mínima obtida por estudantes foi de 4 pontos e a máxima de 7 pontos. Ainda nesta dimensão, a maior parte dos estudantes acertou todas as questões, obtendo pontuação igual a 7 (Tabela 4).

Acerca da dimensão de aspectos epidemiológicos, composta por 4 quesitos, a média da pontuação encontrada entre os estudantes foi 3,10 (DP=0,69), a pontuação mínima obtida pelos estudantes foi 1, e o máximo 4. A pontuação mais observada entre os estudantes, representada pela moda, foi de 3 nesta dimensão (Tabela 4).

Relacionado ao escore total, que representa a pontuação nas duas dimensões e é composto pior 11 quesitos, observou-se uma média de 9,68 (DP-0,98) pontos. A pontuação mínima observada entre os alunos foi de 6 acertos e a máxima de 11 acertos. A pontuação mais frequente entre os estudantes foi de 10 pontos (Tabela 4 ). 
Tabela 4 - Pontuação dos acertos considerando a as dimensões avaliadas e a pontuação total.

\begin{tabular}{lcccccc}
\hline Variáveis & Média & DP & Moda & Mediana & Mínimo & Máximo \\
\hline $\begin{array}{l}\text { Escore: } \quad \text { Aspectos } \\
\text { biológicos e curso clínico }\end{array}$ & 6,57 & 0,66 & 7,00 & 7,00 & 4,00 & 7,00 \\
$\begin{array}{l}\text { da doença } \\
\text { Aspectos }\end{array}$ & 3,10 & 0,69 & 3,00 & 3,00 & 1,00 & 4,00 \\
\hline $\begin{array}{l}\text { Escore: } \\
\text { epidemiológicos }\end{array}$ & & & & & & \\
\hline Escore total & 9,68 & 0,98 & 10,00 & 10,00 & 6,00 & 11,00 \\
\hline
\end{tabular}

Fonte: Própria.

Ao comparar os estudantes que acertaram todos os quesitos, ou seja, obtiveram a pontuação máxima, com os demais estudantes, observou-se que não foram encontradas diferenças estatisticamente significantes entres os cursos avaliados, embora o curso de enfermagem possua menor percentual de estudantes com pontuação máxima (17,2\%) e o curso de nutrição obtiveram o maior percentual (22,2\%). Não foram observadas diferenças estatisticamente significantes entre os estudantes que receberam orientação ou treinamento sobre prevenção e controle da doença, embora os que não receberam tal treinamento tenham um percentual maior de acertos $(22,4 \%)$. Com relação aos meios de informação mais utilizados, diferenças estatisticamente significantes só foram encontradas entre os estudantes que afirmaram usar a internet, onde todos os alunos que acertaram todos os quesitos utilizaram a internet como meio de informação acerca da COVID-19 (Tabela 5). 
Tabela 5 - Análise da diferença entre os estudantes que responderam adequadamente a todos os quesitos, por curso, realização de treinamento e meios de informação utilizados, através do teste do Qui-quadrado de Pearson, Brasil, 2020.

\begin{tabular}{|c|c|c|c|}
\hline \multirow[b]{2}{*}{ Variáveis } & \multicolumn{2}{|c|}{$\begin{array}{l}\text { Estudantes que responderam } \\
\text { adequadamente a todos os } \\
\text { quesitos }\end{array}$} & \multirow[b]{2}{*}{$\begin{array}{l}\text { valor } \\
\text { de } p\end{array}$} \\
\hline & $\begin{array}{l}\text { Sim } \\
\text { n (\%) }\end{array}$ & $\begin{array}{l}\text { Não } \\
\text { n(\%) }\end{array}$ & \\
\hline \multicolumn{4}{|l|}{ Curso } \\
\hline Enfermagem & $10(17,2)$ & $48(82,2)$ & \multirow{4}{*}{0,827} \\
\hline Fisioterapia & $14(17,9)$ & $64(82,1)$ & \\
\hline Nutrição & $18(22,2)$ & $63(77,8)$ & \\
\hline Psicologia & $21(21,6)$ & $76(78,4)$ & \\
\hline \multicolumn{4}{|c|}{$\begin{array}{l}\text { Recebi algum tipo de treinamento ou } \\
\text { orientação sobre prevenção e controle de } \\
\text { infecção pelo coronavírus }\end{array}$} \\
\hline Discordo & $22(22,4)$ & $76(77,6)$ & \multirow{2}{*}{0,477} \\
\hline Concordo & $41(19,0)$ & $175(81,0)$ & \\
\hline \multicolumn{4}{|c|}{$\begin{array}{l}\text { Meios de informação mais utilizados sobre } \\
\text { coronavírus: }\end{array}$} \\
\hline \multicolumn{4}{|l|}{ Internet } \\
\hline $\operatorname{Sim}$ & $63(21,9)$ & $225(78,1)$ & \multirow{2}{*}{$0,008^{*}$} \\
\hline Não & $0(0,0)$ & $26(100,0)$ & \\
\hline \multicolumn{4}{|l|}{ Redes sociais } \\
\hline Sim & $21(19,4)$ & $87(80,6)$ & \\
\hline Não & $42(20,4)$ & $164(79,6)$ & \\
\hline \multicolumn{4}{|c|}{ Artigos científicos } \\
\hline Sim & $28(20,9)$ & $106(79,1)$ & \multirow{2}{*}{0,751} \\
\hline Não & $35(19,4)$ & $145(80,6)$ & \\
\hline \multicolumn{4}{|c|}{ Amigos e familiares } \\
\hline Sim & $15(21,7)$ & $54(78,3)$ & \multirow{2}{*}{0,694} \\
\hline Não & $48(19,6)$ & $197(80,4)$ & \\
\hline \multicolumn{4}{|l|}{ Outros } \\
\hline Sim & $22(22,9)$ & $74(77,1)$ & \multirow{2}{*}{0,402} \\
\hline Não & $41(18,8)$ & $177(82,1)$ & \\
\hline
\end{tabular}

Fonte: Própria. 


\section{DISCUSSÃO}

A partir dos resultados obtidos por esse estudo, foi possível observar que os estudantes detêm algum tipo de conhecimento e informação sobre a pandemia do covid-19, classificado como regular quanto aos aspectos gerais da doença, porém precário quando se trata dos aspectos epidemiológicos. A maior parte dessas informações é recebida mediante o acesso à internet, e este meio se apresentou como um fator significante para acertar todos os quesitos.

No território brasileiro, a maior parte da população já tem acesso à internet graças a rápida evolução das Tecnologias de Informação e Comunicação (TIC). É preciso considerar que o fato de serem estudantes universitários, pode ser um indicativo de que a maior parte possua acesso em sua casa. Todavia, estudo feito para avaliar conhecimento, atitudes e prática de estudantes residentes na China sobre o covid-19 enfatiza que as populações mais vulneráveis tendem a possuir acesso à internet limitado ou nem possuir; pessoas mais velhas e residentes de áreas rurais são mais propensas a ter pouco conhecimento devido ao acesso precário, e isso pode resultar em atitudes negativas e práticas preventivas inadequadas em direção a Covid-19 (ZHONG et al., 2020).

Um fator preocupante é a identificação de que a busca de informações em artigos científicos foi efetuada por apenas $42,7 \%$ dos estudantes. As redes sociais foi o meio mais acessado. Sabendo que a graduação é o período de construção da carreira profissional, é necessário estimular que os alunos façam a atualização constante através das produções científicas, como garantia de maior critério de qualidade das informações quando comparadas as redes sociais.

No que diz respeito a receber algum tipo de treinamento e orientação sobre prevenção e controle do COVID-19, os estudantes de graduação em Psicologia, seguido dos de Nutrição foram os que mais disseram não ter recebido nenhum tipo de orientação, embora, a maior parte dos participantes desta pesquisa tenha referido algum tipo de orientação ou treinamento recebido. Além disso, também foi questionado acerca do conhecimento sobre a utilização que Equipamento de Proteção Individual como método para evitar a infecção pelo coronavírus, onde a maior proporção da amostra referiu conhecer, sendo os menos conhecedores os alunos de psicologia seguido do curso de fisioterapia. Diversas universidades públicas brasileiras estão fornecendo cursos com o objetivo de orientar estudantes e 
profissionais da saúde sobre o coronavírus, a exemplo da Universidade Federal do Rio Grande do Norte que em parceria com o Ministério da Saúde já possui um curso on-line aberto para todo o Brasil (https://avasus.ufrn.br/local/avasplugin/cursos/curso.php?id=327). No entanto, apesar de ser gratuito e existir ampla divulgação sobre os cursos, pode existir uma falta de interesse por parte dos acadêmicos em buscar essas informações.

Ainda assim, é preciso enfatizar que até 27 de abril de 2020, nenhum tratamento definitivo para cura foi encontrado, o que reforça a necessidade do conhecimento a respeito das formas de prevenção da doença. Na ausência de tratamentos eficazes, a melhor maneira de lidar com a pandemia do SARS-CoV-2 é a prevenção com objetivo de controlar as fontes de infecção. Para os indivíduos, medidas de proteção, incluindo melhoria da higiene pessoal e de alimentos, uso de máscaras cirúrgicas, utilização de álcool 70\% para desinfecção de sujidades das mãos, descanso adequado e manutenção da sala bem ventilada, podem efetivamente prevenir a infecção por Covid-19 (GUAN et al., 2020).

A maioria dos respondentes afirmou que a interrupção das atividades acadêmicas iria afetar seu rendimento acadêmico e que sentem sua saúde mental prejudicada devido a pandemia. A Organização das Nações Unidas para a Educação, a Ciência e a Cultura (UNESCO) estimou que o fechamento de instituições de ensino devido a pandemia afeta cerca de 890 milhões e estudantes em todo o mundo, numa estimativa de 114 países (UNESCO, 2020). Segundo o monitoramento da UNESCO, mais de 160 países implementaram fechamentos em todo o país, o que impactou mais de $87 \%$ da população estudantil do mundo.

O calendário acadêmico da UFRN está suspenso desde o dia 17 de maio de 2020, e nessa perspectiva, o ensino on-line tornou-se uma nova rotina para algumas instituições. Considerando que o ensino à distância $(\mathrm{EaD})$ apresenta desafios significativos no estado do Rio Grande do Norte, já que pela desigualdade social existente nem todos os alunos têm acesso a esse tipo de ferramenta educacional, o EaD não foi efetivado como uma opção para a continuidade das aulas nessa instituição. Universidades de todo o mundo não sabem ao certo quanto tempo durará a crise do coronavírus e como isso pode afetar a saúde mental de estudantes e professores (ARAÚJO et al., 2020).

Especialistas alertam que o impacto do coronavírus irá repercutir no ensino superior global por muito mais tempo mesmo após a pandemia ser controlada. Nas áreas mais afetadas, 
as universidades enfrentam a perspectiva de perder até um semestre inteiro (THE WORLD UNIVERSITY RANKS, 2020). Assim, estudos já mostram que a ansiedade e a depressão, emergem pelas incertezas e intensificação do fluxo de informações. Consequências fisiológicas negativas do estresse podem se manifestar, para os alunos podem se sobressair, a solidão, que aumentará nessas circunstâncias, tendo um impacto negativo na educação e, portanto, na dor e no sofrimento psicológicos (ARAÚJO et al., 2020)..

Quando avaliado os aspectos biológicos e curso clínico da doença, foi possível identificar que de modo geral, os estudantes possuem um conhecimento regular quanto a esse domínio. No que concerne ao conhecimento do nome científico, a origem do Coronavírus e os sinais e sintomas da doença, a maior parcela afirmou ter esse conhecimento. Em estudo semelhante realizado no Paquistão com profissionais, professores e alunos da saúde, referiu que dentro desse grupo amostral, 95,36\% conheciam o nome científico e $82,45 \%$ dos entrevistados disseram conhecer sua origem. Já em relação aos sinais e sintomas da doença, identificou-se resultados semelhantes nesses questionamentos, no entanto, profissionais médicos apresentaram um conhecimento maior quando comparado com os demais grupos, indicando que o nível de consciência está vinculado à profissão de um indivíduo (KHAN et al., 2020).

No presente estudo não foi identificada diferença significativa entre os cursos de graduação para as variáveis pesquisadas, no entanto, alguns estudantes do curso de Nutrição seguido de Psicologia apontaram de forma equivocada a maneira de identificar um indivíduo infectado, afirmando que "ter febre, gripe e tosse significa que o indivíduo está infectado". Estudos já apontam que os sinais e sintomas desse agravo vão além, e seus principais sintomas clínicos incluem febre, tosse seca, fadiga, mialgia e dispneia. Na China, 18,5\% dos pacientes com Covid-19 evolui para o estágio grave, que é caracterizada por síndrome do desconforto respiratório agudo, choque séptico, acidose metabólica de difícil tratamento, e disfunção de sangramento e coagulação (ZHONG et al., 2020). Para que se afirme que o indivíduo realmente está infectado, é necessário realizar além da avaliação clínica, a realização de testes sorológicos necessários para que se confirme a infecção.

O contágio por animais de estimação ainda não foi descrito na literatura, sendo uma hipótese até então eliminada, nesse sentido é possível perceber que os alunos possuem conhecimento adequado neste quesito. De modo semelhante, maior parte concordou que 
nem todos evoluirão para casos graves. Sabe-se que muitos casos irão desenvolver sintomas leves, e outros podem inclusive serem portadores assintomáticos, dessa forma, aponta-se a necessidade de reforçar o conhecimento desses estudantes acerca das estratégias de prevenção de contágio, isolamento e tratamentos de suporte e manutenção de ordens sociais (GUAN et al., 2020) $)^{13}$.

Avaliando a dimensão dos aspectos epidemiológicos, considera-se um nível de conhecimento precário nesse domínio, considerando a média de acertos quando comparado ao anterior. Embora não tenha havido diferença significativa entre os cursos, é importante enfatizar a necessidade de potencializar o ensino da Epidemiologia e outros componentes curriculares correlatos para o ensino na graduação. Os estudos epidemiológicos exercem grande influência sobre a saúde (ROTHMAN; GREENLAND; LASH, 2020) assim, essa disciplina constitui-se com um pilar fundamental para formação do aluno da área da saúde, nela, é possível entender os fenômenos existentes em pandemias como a do Covid-19, por isso, reforça-se a necessidade de abordagens metodológicas que possam garantir o conhecimento adequado do aluno.

Destacamos que os alunos concordam com as medidas de distanciamento social. À época dessa pesquisa, os governantes estaduais decretaram essa medida como forma de evitar a propagação do contágio do Covid-19, como meio de evitar que as pessoas tenham contato direto. Para incentivar essa estratégia de controle, ocorreu o fechamento de escolas, universidades, comércios não essenciais, áreas públicas de lazer e outros estabelecimentos (HALE et al., 2020). Grande parte da população brasileira aderiu ao isolamento social, entendendo que para o momento essa é a medida mais eficaz para prevenção e atenuação da curva de contágio no país (GARCIA; DUARTE, 2020).

\section{CONCLUSÃo}

O conhecimento e acesso às informações dos estudantes de graduação sobre o Covid19 pode ser considerado regular, o que alerta acerca da importância do acompanhamento das informações bem como das atualizações diárias sobre a doença, pois, enquanto futuros 
profissionais de saúde precisam incorporar na sua rotina a aquisição de informações relevantes e de qualidade sobre os temas atuais da saúde. Os dados coletados nesse estudo podem estar sujeito ao viés de seleção, apesar de estarem dentro dos parâmetros amostrais, e podem não caracterizar a UFRN ou outras universidades públicas. Porém, os dados aqui apresentados são úteis para representar a situação dos alunos da FACISA e fornecer informação para que os gestores desta unidade acadêmica possam orientar ações de qualificação direcionadas aos alunos.

\section{REFERÊNCIAS}

ARAÚJO, F.J.O; LIMA, L.S.A; CIDADE, P.I.M; NOBRE, C.B; NETO, M.L.R. Impact Of Sars-Cov-2 And Its Reverberation In Global Higher Education And Mental Health. Psychiatry Research, p. 112977, 2020. Disponível em: < https://www.sciencedirect.com/science/article/pii/S0165178120307009?casa_token=GK5sJjctxEQAAAAA:689a 3iGCuPOZUynoOcd-j9nc_RZfj_DAtCckCC5iW9v42QsvpAsy3gFH1FqNIbohggeB_7ARSUo>. Acesso em: 05 mai. 2020.

BHAGAVATHULA, A.S; ALDHALEEI, A; RAHMANI, J; MAHABADI, M.A; BANDARI, D.K. Novel Coronavirus (COVID19) Knowledge and Perceptions: A Survey on Healthcare workers. medRxiv, 2020. Disponível em: < https://www.medrxiv.org/content/medrxiv/early/2020/03/16/2020.03.09.20033381.full.pdf>. Acesso em: 05 mai. 2020.

CRODA, J; OLIVEIRA, W.K.D; FRUTUOSO, R.L; MANDETTA, L.H; SILVA, D.C.B; SOUSA, J.D.B; LACERDA, M.V.G. COVID-19 in Brazil: advantages of a socialized unified health system and preparation to contain cases. Revista da Sociedade Brasileira de Medicina Tropical, v. 53, 2020. Disponível em: $<$ https://www.scielo.br/scielo.php?pid=S0037-86822020000101000\&script=sci_arttext>. Acesso em: 05 mai. 2020.

DONG, E; DU, H; GARDNER, L An interactive web-based dashboard to track COVID-19 in real time. The Lancet infectious diseases, v.20, n.5, p.533-534, 2020. Disponível em: < https://www.thelancet.com/journals/laninf/article/PIIS1473-3099(20)30120-1/fulltext>. 05 mai. 2020.

GARCIA, L.P; DUARTE, E. Intervenções não farmacológicas para o enfrentamento à epidemia da COVID-19 no Brasil. Epidemiologia e Serviços de Saúde. v.29. n.2, 2020.29:2.

GELDSETZER, P. Use of Rapid Online Surveys to Assess People's Perceptions During Infectious Disease Outbreaks: A Cross-sectional Survey on COVID-19. Journal of medical Internet research, v. 22, n. 4, p. e18790, 2020. Disponível em: < https://www.jmir.org/2020/4/e18790/>. Acesso em: 05 mai. 2020.

GUAN, W; NI, Z.Y; HU, Y; LIANG, W.H; OU, C.Q; HE, J.X; DU, B. Clinical characteristics of 2019 novel coronavirus infection in China. MedRxiv, 2020. Disponível em: https://www.medrxiv.org/content/10.1101/2020.02.06.20020974v1>. Acesso em: 05 mai. 2020.

HALE, T; WEBSTER, S. Oxford COVID-19 government response tracker. Disponível em: <Erro! A referência de hiperlink não é válida.>. Acesso em: 05 mai. 2020. 
KHAN, S; KHAN, M; MAQSOOD, K; HUSSAIN, T. E; ZEESHAN, M. Is Pakistan prepared for the COVID-19 epidemic? A questionnaire-based survey. Journal of Medical Virology, 2020. Disponível em: < https://onlinelibrary.wiley.com/doi/full/10.1002/jmv.25814>. Acesso em: 05 mai. 2020.

LAI, C.C; SHIH, T.P; KO, W. C; TANG, H. J; HSUEH, P.R. Severe acute respiratory syndrome coronavirus 2 (SARSCoV-2) and corona virus disease-2019 (COVID-19): the epidemic and the challenges. International journal of antimicrobial agents, p. 105924, 2020. Disponível em: < https://www.sciencedirect.com/science/article/pii/s0924857920300674?casa_token=QFypNn95N2AAAAAA:zb Id0eCMZISJtdgCHc3FaQb9r4QlPOPBQWaS5sRrkDHRgqXiVcoxFKERDXbRf7eEsv0dG0JQDrQ?. Acesso em: 05 mai. 2020.

LI, Q; GUAN, X; WU, P; WANG, X; ZHOU, L; TONG, Y; XING, X. Early transmission dynamics in Wuhan, China, of novel coronavirus-infected pneumonia. New England Journal of Medicine, v.382, p.1199-1207, 2020. Disponível em: < https://www.nejm.org/doi/full/10.1056/NEJMoa2001316>. Acesso em: 05 mai. 2020.

ROTHMAN, K; GREENLAND, S; LASH, T. Epidemiologia Moderna-3ª Edição. Artmed Editora. 2016.

Secretaria de Saúde Pública do Estado do Rio Grande do Norte. Boletim Epidemiológico. 2020. Disponível em: <http://www.saude.rn.gov.br/Conteudo.asp?TRAN=ITEM\&TARG=230053\&ACT=\&PAGE=\&PARM=\&LBL=NOT\%C DCIA10>. Acesso em: 28 abr. 2020.

THE WORLD UNIVERSITY RANKINGS, 2020. Universities brace for lasting impact of coronavirus outbreak. Disponível em: <https://www.timeshighereducation.com/news/universities-brace-lasting-impactcoronavirus-outbreak>. Acesso em: 05 mai. 2020.

UNESCO, 2020. COVID-19 educational disruption and response. 2020. Disponível em: $<$ https://en.unesco.org/themes/education-emergencies/coronavirus-school-closures>. Acesso em: 05 mai. 2020.

WORLD HEALTH ORGANIZATION. Coronavirus disease 2019 (COVID-19). Situation Report - 98. Disponível em: $<$ https://www.who.int/docs/default-source/coronaviruse/situation-reports/20200427-sitrep-98-covid19.pdf?sfvrsn=90323472_4>. Acesso em: 05 mai. 2020.

WORLD HEALTH ORGANIZATION. Novel coronavirus (COVID-19) situation. Disponível em: <https:// https://experience.arcgis.com/experience/685d0ace521648f8a5beeeee1b9125cd>. Acesso em: 05 mai. 2020.

YIN, Y; WUNDERINK, R.G. MERS, SARS and other coronaviruses as causes of pneumonia. Respirology, v. 23, n. 2, p. 130-137, 2018. Disponível em: < https://onlinelibrary.wiley.com/doi/full/10.1111/resp.13196>. Acesso em: 05 mai. 2020.

ZHONG, B.O; LUO, W; LI, H.M; ZHANG, Q.Q; LIU, X.G; LI, W.T; LI, Y. Knowledge, attitudes, and practices towards COVID-19 among Chinese residents during the rapid rise period of the COVID-19 outbreak: a quick online crosssectional survey. International Journal of Biological Sciences, v. 16, n. 10, p. 1745, 2020. Disponível em: < https://www.ncbi.nlm.nih.gov/pmc/articles/PMC7098034/>. Acesso em: 05 mai. 2020.

ZHU, N; ZHANG, D; WANG, W; LI, X; YANG, B; SONG, J; NIU, P. A novel coronavirus from patients with pneumonia in China, 2019. New England Journal of Medicine, n.382, p.727-733, 2020. Disponível em: < https://www.nejm.org/doi/full/10.1056/NEJMoa2001017>. Acesso em: 05 mai. 2020.

\section{(c) $\mathbf{E r}$}

Este trabalho está licenciado com uma Licença Creative Commons - Atribuição 4.0 Internacional. 